\title{
Translation Initiation Abnormality
}

National Cancer Institute

\section{Source}

National Cancer Institute. Translation Initiation Abnormality. NCI Thesaurus. Code C45635.

An abnormality that results from a functional change in the mRNA sequence at the translation initiation codon. In eukaryotic organisms, the initiation codon is always AUG. A mutation in either DNA or corresponding mRNA that alters this initiation sequence causes termination of mRNA translation. No functional protein product is synthesized when a translation initiation abnormality exists. 